\title{
Research on Security Traceability Platform of Agricultural Products Based on Internet of Things
}

\section{Lihua Jiang ${ }^{1, a^{*}}$ and Kaiqiong Sun ${ }^{2, b}$}

\author{
${ }^{1}$ School of Computer,Wuhan Polytechnic University,Wuhan Hubei, 430023,China \\ ${ }^{2}$ School of Computer,Wuhan Polytechnic University, Wuhan Hubei, 430023, China \\ ajianglihua@whpu.edu.cn, b40473153@qq.com \\ * the corresponding author
}

Keyword: Trace; Collection; Internet of things; Monitor; Sensor

\begin{abstract}
In order to meet the needs of modernization, intelligence and informatization of agricultural products, the agricultural product security traceability platform is proposed, and a complete set of agricultural product data sensing network is established. Through the design of a data acquisition gateway for agricultural products, the real-time automatic collection, processing and display of the parameters such as illumination, $\mathrm{PH}$ value, and dissolved oxygen content in water are realized, the fast and accurate data transmission between sensor detection nodes and coordinator nodes is completed, and the real-time remote monitoring is carried out. The platform mainly includes agricultural product safety monitoring and sensor data acquisition, Internet of things software architecture, etc. The results show that it has obvious effect on improving the agricultural products traceability efficiency and reducing the tracking and monitoring costs of agricultural products.
\end{abstract}

\section{Introduction}

IOT that is Internet Introduction of Things can put any objects connected with the Internet, make information exchange and communicate, in order to realize a network to the intelligently identification, location, tracking and monitoring ${ }^{[1]}$. In traditional agricultural method, people look for farmland information is limited, mainly through the artificial measure, which spends a lot of manpower, poor real-time and low productive efficiency. Through the IOT, people can collect realtime temperature, humidity, light intensity and dissolved oxygen content, and such various parameters, so as to make scientific forecast and real-time control ${ }^{[2]}$.

The application based on the safety traceability technology of Internet of things, each agricultural product is stuck with two-dimension code of safety information, replace or replenish existing onedimension codes on the fresh agricultural products packaging, after consumers buy the agricultural product which attached to two-dimension $\operatorname{coder}^{[3]}$, they can look for the agricultural product's safety information through the two-dimension code which is provided by the platform ${ }^{[4]}$.

\section{Research Status and Related Technology}

Although in the construction aspect of traceability system ,our country has made great achievements, there are still some problems, mainly included the following aspects: although food production enterprises is very familiar with their business process, the information's level is usually low, they can not build a higher level of information food safety traceability system by their own strength. It is predicted that in 2015, the market scale of food safety traceability system is about 8.5 billion $^{[5]}$.

The tracing system for agricultural products has excellence response in the industry and its technical level stays not only leading position in domestic industry but also in the world at the leading level, the products in the food industry has been applied widely, it occupies a significant share of the market, thus it has a strong competitive advantage.

Agricultural product safety tracing system can be widely used in the safety of agricultural, forest, and fishery food and it applies to the livestock poultry food, fruits and vegetables aquatic food and 
so on. Through the implementation of tracing system can inquire the source circulating information in the market, to find person in charge of the food quality, to force the security risks of enterprises out of the market, however, enterprises with good quality can also build credibility ${ }^{[6]}$.

The application of quality and safety tracing system has the significant meaning and value for both the enterprise and society. For the enterprise, the tracing system can solve the quality problem in the process of its production and management. For the society, the wide application of the tracing system can effectively realize the product quality supervision, and prevent the product quality problem $^{[7]}$.

\section{The Data Acquisition and Processing of Agricultural Product}

The Information of Agriculture Product's Acquiring and Transmission. Agricultural enterprises get the data information of transmission by placing all kinds of sensors in different locations in the producing base.After building the detection network, the sensor will automatically collect the surrounding information such as temperature, humidity, $\mathrm{PH}$ value, the amount of dissolved oxygen, light and so on. Meanwhile, the collected environment information will be sent to the platform through the network. The data of Agricultural monitoring topology is shown in Fig. 1.

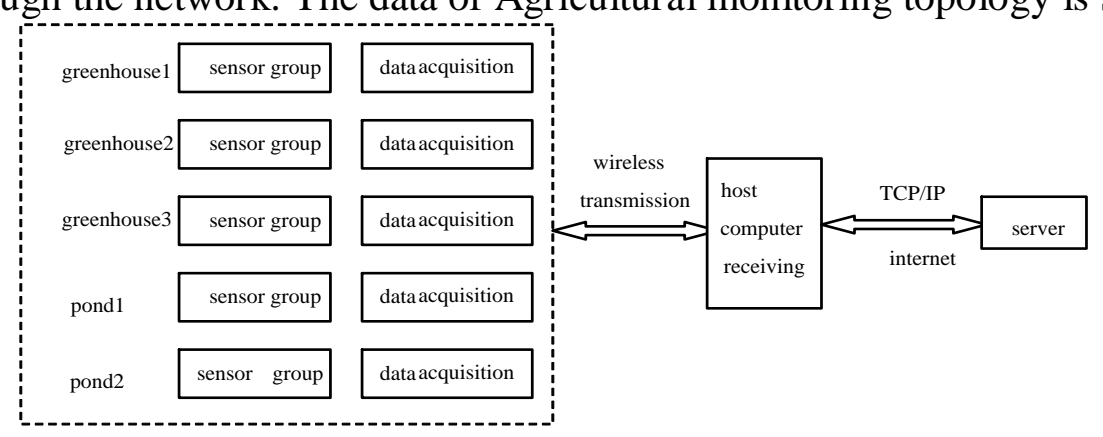

Figure 1. The data of Agricultural Monitoring Topology

Agricultural habitat sensing information is an important part in modern intellectual sense technology and is the most critical part. To develop a monitoring machine which is suitable for designing the fish ponds niche information dynamic with the safe update, including humidity, temperature, $\mathrm{pH}$ value, dissolved oxygen illumination sensor, serial conversion equipment[8].

The information of agricultural inspecting includes: temperature, humidity, light, heavy metals and pesticide residues, and the heavy metals and pesticide residues need to be detected after sampling. Pool information detection includes: temperature, light, transparency, $\mathrm{pH}$ value, etc.

Environmental monitor uses wireless intelligent sensor JZH-013 and JZH-003, JZH-013. JZH013 mainly monitors soil temperature, the sensor based on ZigBee protocol, output data is the Modbus protocol format. The system will prepare to develop an internet gateway software, through monitoring computer serial port sensor data, and to collected data it can filtering, parsing, format conversion and data transmission etc.

Gateway Design of Agricultural Product Information Acquisition. The template is used to format your paper and style the text. All margins, column widths, line spaces, and text fonts are prescribed; please do not alter them. You may note peculiarities.

The Internet of things gateway is used to realize the insert and access of Internet of things, and provide mostly the middle ware services, including ONS service support, for analyzing the network position and data storage location of the node EPC of the Internet of things. The Internet of things gateway can be used as a module in the controller of the PC, through the controller's proprietary network, the collected data will be sent to the data center ${ }^{[9]}$.

Data Transmission. The hardware infrastructure will transmit the collected information on various types of networks, which includes gateway equipment and various types of cable or wireless network. Multiple terminal nodes will collect the processing information through the sink node, then the star network converge will get the gateway node, making data fusion ${ }^{[10]}$. 
Data Filtering. As a kind of non-contact automatic recognition technology, RFID brings the problem of data redundancy as the ability of automatic and real-time data acquisition atthe same time.. It is caused by data redundant including: and it is in the short term with a reading and writing device on the same label data repeated submission; multiple approaches to read and write to these data are reported, and some other systems do not care about the data are reported.

Data Integration and Format Conversion. The raw RFID data streams from read-write device are some simple scattered single information. It also needs to convert the data format of the acquisition to the data format which is understandable to the application system, and transmit the data to the back-end application system.

Analysis Maintenance of Agricultural Product Information. Operating platform need ONS(Object Naming Service) of the service support for analysis networking nodes of EPC network location and the data storage. Read data pass to background system through network connection.

\section{The Agricultural Product Traceablity Platform Scheme}

Taceability Platform Framework Component. Define abbreviations and acronyms the first time they are used in the text, even after they have been defined in the abstract. Abbreviations such as IEEE, SI, MKS, CGS, sc, dc, and rms do not have to be defined. Do not use abbreviations in the title or heads unless they are unavoidable.

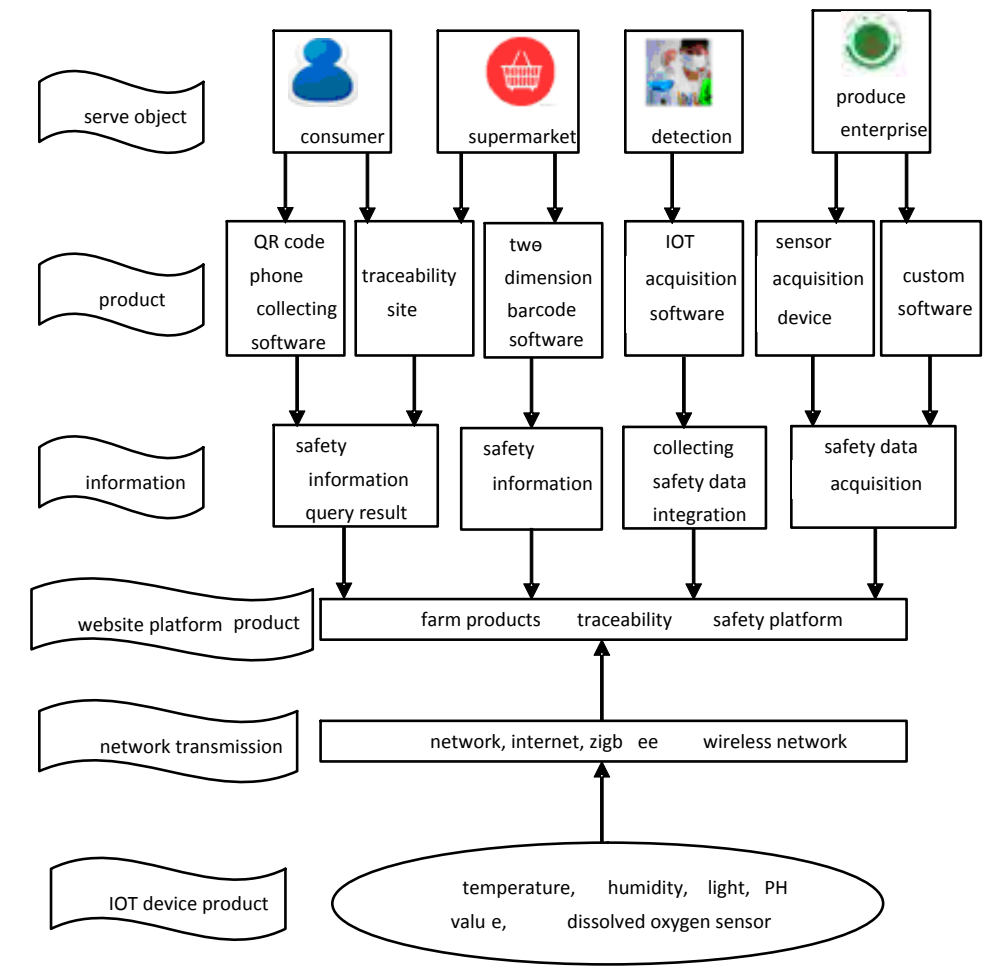

Figure 2. Traceability platform framework

Agricultural product security traceability platform framework is shown in Fig. 2 [11].

Agricultural Product Traceability System Architecture. Agricultural product safety traceability platform based on IOT, and by the technology of perception collecting front-end, application service layer as background support, the system architecture is shown in Fig.3, mainly including Internet of things sensing layer, network layer and application service layer.

Sensing layer which is mainly achieves the smart sense function, the part of the sensor uses all kinds of sensor device to collect the data of cultivation, fertilization, pesticide, as well as including acquisition, capture and object identification. Which will get the required information in large-scale, long-term including product categories, fertilizer name, feeding time and feeding density. It is also necessary to solve the technical problems such as signal type, transmission interface, data format inconsistency and so on. 


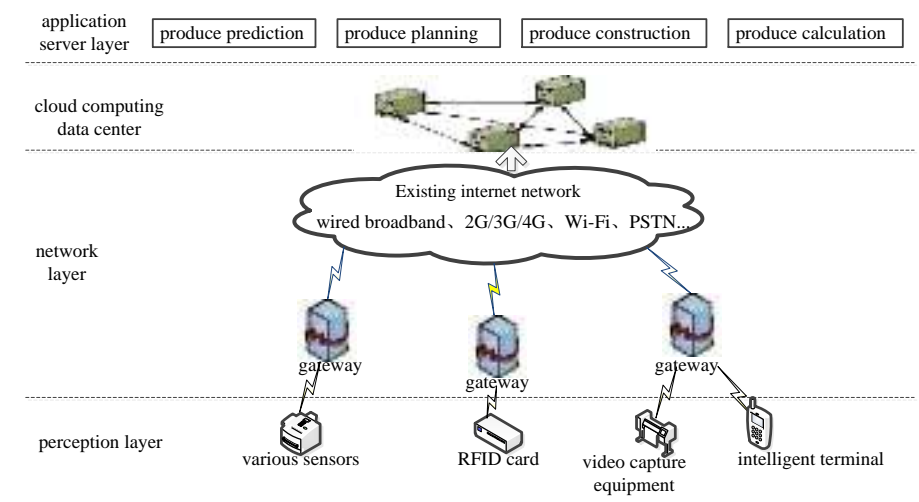

Figure 3. Traceability architecture of agricultural product

Network layer is mainly to achieve information transmission and communication based on the existing Internet, combined with wired broadband, mobile $2 \mathrm{G} / 3 \mathrm{G}$ network, WiFi, Bluetooth and other transmission technology, to achieve the interconnection and cooperative work of the heterogenous network.

Application service layer is based on the network layer, which process the collection data, and which transform digital signal, that these messages should be searched and monitored according to user requirement.

The Process of Using This Platform. Consumers through the cell phone barcode software of agricultural products can inquire the quality and safety, logistics information as well as the cultivating information of the agricultural products .enterprise of agricultural products and supermarket can demand the results through the phone and website and this software can allow inspector and enterprises of agricultural products can update the security information through website, barcode software and acquisition software.

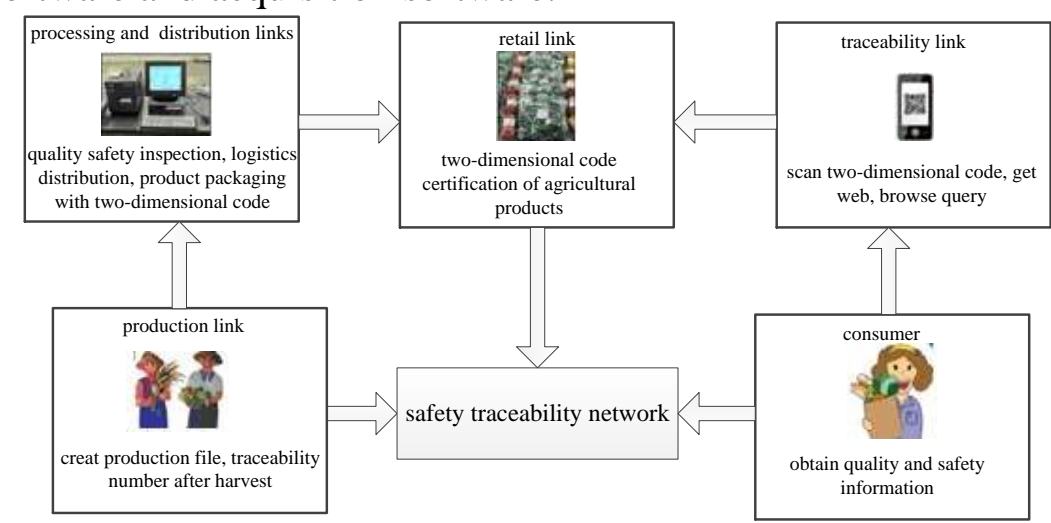

Figure 4. Agricultural products quality and safety traceability process

During the production planting, for the agricultural product establishing the production chain code (or produce batch) and manufacturing file. (it could also say the QRcode or RFID), car loading after the information affirmed, in this way, the tracing system can be assured combined with the batch and planter identification.

During the processing(two-dimension code), choosing agricultural products information from the system to the processing, after reading successfully the label, recording the batch of real-time processing of agricultural products. First it reads QRcode information of agricultural products, and then test results of agricultural products, processing unit, the processing date of information, as well as the related information such as packing weight adding to the QRcode.

During the shipping, in the procession of transportation and distribution, vehicle can identify the tag, a record of license plates, drivers and products information, which transport the goods to the appointed warehouse.

During the consumption, supermarket identifies the electronic label information on the product package from the processing enterprise, the terminal retailer updates the inventory information. they 
can query the information according to the traceability code login traceability system, realize the product traceability.

\section{Conclusion}

Agricultural product safety traceability platform which is based on Internet of things study the data collection, processing, sorting, transmission, analysis and release, query and trace, such as a full range of products, which is proposed based on the quality and safety of agricultural products traceability of low cost, high efficiency, the whole chain, a comprehensive solution. Flexible and extensible of produce quality safety traceability system architecture, compatible with different areas and different industry segment, adapt and inclusion differentiation of enterprise and product information coding system trace levels, for consumer, business and government to provide interdisciplinary, cross platform of public service.

\section{Acknowledgement}

This work was supported by the Natural Science Foundation of Hubei Province of China under Grant No.2016CFB470.

\section{References}

[1] LIU Yu-xiao etc. EDA technology and VHDL circuit development application practice[M], Beijing:The Press of electronics,2009.

[2] LUO Li-fan etc.FPGA development based on VHDL induction quickly, skill and instance[M], Beijing:The Press of posts \&telecom,2009.

[3] LIU Zhao-han. VHDL chip design[M], Beijing:The Press of Tsinghua University, 2006.

[4] XIN Jian-pin,ZENG Fan-tai.VHDL Programming tutorial (the third edition) [M], Beijing:The Press of Tsinghua University, 2005.

[5] WANG Xu-ying,LU Ying-hua,ZHANG Li-kun.Design and Implemention of high-speed realtime data acquisition system based on FPGA[J].The Jounal of China Universities of Posts and Telecommunicaions, 2008,13(4):61-66.

[6] Ma Qungang,Yang Yintang,Li Yuejin.New Design Method for the Optimal Energy Delay Product of FPGA Based on the Interconnect[J] .Journal ofXidian University , 2009 ,31(1): 3235.

[7] Wang P,Franca JE.Multirate switched-capacitor circuits for 2-D signal processing[M].Kluwer Acadmic Publishers, 1998.

[8] IEEE Standard VHDL Language Reference Manual[S]. IEEE SUT,2009,1067-1987.

[9] ROMAN L.FANK V A Study of the speedups and competitiveness of FPGA Soft Processor Cores using Dynamic Hardware/Software Partitoning,2010.

[10] Volnei A.Pedroni-Circuit Design with VHDL[M].MIT Press,2009.

[11] CHENG C C,HUANG C T.Multiple-lifting scheme:memory-efficent VLSI implementation for line-based 2-D DWT:Proceedings of International Symposium on Circuits and Systems, Kobe, Japan, May,2005[C].Kobe,2009:23-26. 